\title{
Tetrapolar bioimpedance measurements compared to four-wire resistance measurements
}

\author{
Ramon Pallàs Areny \\ Universitat Politècnica de Catalunya, BarcelonaTech (UPC), Barcelona, Spain \\ E-mail any correspondence to: ramon.pallas@upc.edu
}

It is often (wrongly) assumed or implied that tetrapolar bioimpedance measurements convey the same advantage than the four-wire resistance measurement method proposed by Lord Kelvin, namely, minimizing the influence of the leads that connect the resistance under test (RUT) to the measurement instruments, which is mostly beneficial in low-value resistance measurements. Tetrapolar bioimpedance measurements reduce, rather than minimize, the effect of electrode impedances but they suffer from concomitant undesired effects mainly attributable to moving from: 1) DC to AC measurements, 2) electronic to electrolytic conductors, 3 ) resistance to impedance measurement, 4) single-ended to differential voltage measurements, and 5) electronic components or devices to volume conductors.

Moving from two-wire to four-wire resistance measurements implies a measurement setup with two circuits, one for current injection and the other one for voltage detection, instead of a single circuit where the current source and the voltmeter share the two leads that connect them to the RUT. Having two close circuits is not a major problem in DC measurements but AC currents generate variable magnetic fields that induce voltages in any conductive loop linked by these fields. Therefore, the area of the injecting and detecting circuits in four-wire AC measurements should be minimized otherwise the voltage that the current injection circuit would directly induce in the voltage detection circuit would add up to the drop in voltage across the RUT. Furthermore, the stray capacitance between the injection and the detection leads will result in displacement currents coupled to the detection circuit. The drop in voltage across the RUT because of these displacement currents will increase with the resistance being measured. As a result, the apparent resistance measured may depend on frequency well before the stray capacitance between the resistor terminals affects the result. Therefore, single leads in the detection (or injection) circuit may need to be replaced by coaxial cables, which will add stray capacitances to ground hence limiting the maximal measurement frequency. Finally, whereas in DC measurements the high input resistance of the voltmeter implies a very small measurement current hence a negligible voltage drop across the leads in the detection circuit, the input impedance of any AC voltmeter decreases when frequency increases. Consequently, the voltage loading effect will increase with frequency and the apparent $A C$ resistance will decrease.

Current in electrolytic conductors is carried by ions. Hence, to connect them to electronic circuits we use electrodes, which usually involve a Faradaic reaction (charge transfer) on the surface of the electronic conductor in contact with the electrolyte. Capacitive electrodes with an insulator between the electronic conductor and the electrolyte, hence no charge transfer, are also feasible but their high impedance poses a great challenge to instrumentation electronics. In any case, electrode impedances are often larger than the impedance under test (ZUT), and this is one of the main reasons to use four electrodes instead of two electrodes. But the scenario is quite different from that found in low-value resistance measurements because current in the detection circuit in $\mathrm{AC}$ measurements will be relatively large due to the finite input impedance of the voltage meter hence the voltage drop across each of the two voltage detection electrodes 
can be significant. The measurement deviation will increase with ZUT value because of the larger current through the voltmeter, whereas in DC resistance measurement of electronic conductors the measurement deviation due to contact resistance decreases for large RUT values. This effect may explain, for example, why the apparent resistance of a resistive material or solution decreases for increasing frequency.

$A C$ resistance measurements can benefit from the fact that only voltages in-phase with the excitation are of interest. This allows, for example, the rejection of inductive and capacitive interference by using coherent detection. Impedance measurements that involve determining the real and imaginary impedance components become very difficult when one of them largely predominates. Four-wire measurements are not of much help in this case.

Four-wire and tetrapolar measurements that involve differential voltages complicate the previous problem because the output of differential voltage amplifiers is contributed by both the differential-mode and the common-mode input voltages, according to the respective gains. It turns out that whereas the differential gain is fully specified, or otherwise it can be easily measured, the common-mode gain is seldom specified and it is more difficult to measure. Instead, the modulus of the ratio between the two gains, the so-called, common-mode rejection ratio (CMRR) is given. This CMRR, however, does not inform us about the phase characteristic of the common-mode gain hence does not allow us to calculate the actual contribution of the input common-mode voltage to the output. This effect of the common-mode gain reveals as a change in the apparent impedance when the current injected (or voltage applied) to the ZUT is increased because this increases the common-mode voltage.

Finally, moving from measurements in components or devices to measurements in volume conductors adds at least two more problems: determining the sampled volume and minimizing stray capacitances to ground. Equivalent circuit models and phantoms emulating ZUT can easily lead to the wrong concept that in tetrapolar impedance measurements involving a linear array of electrodes $A, B, C$, and $D$, if, say $A$ and $D$ inject current and $B$ and $C$ measure voltage, the sampled volume is that between $B$ and $C$. It has been theoretically and experimentally demonstrated that this is not the case and that some volumes between $A$ and $D$ contribute to the measured impedance with positive sensitivity whereas other contribute with negative sensitivity or do not contribute at all. Therefore, the difference between the bipolar impedance measured between $A$ and $D$, and the tetrapolar impedance measured between $B$ and $C$ (with an ideal voltmeter) when injecting between $A$ and $D$ is not just because of the reduced influence of electrode impedance and the shorter distance between $B$ and $C$ as compared with the distance between $A$ and $D$.

Stray capacitances to ground, which increase with sample volume and electrode size, will drain current that will increase with frequency. This can result in apparent capacitive or inductive impedance components, depending on the injection-detection strategy, even in bipolar measurements. If stray capacitances from electrodes to ground become significant, tetrapolar bioimpedance measurements can be more affected by those stray capacitances than bipolar measurements.

In summary, tetrapolar bioimpedance measurements cannot be simply thought of as the "AC version" of the fourwire Kelvin method of resistance measurement that "minimizes" the effects of electrodes. There is certainly ample evidence that tetrapolar bioimpedance measurements can reduce the effect of electrode impedance but overlooking the above-mentioned problems may lead to wrong results, misinterpretations, or both. 